\title{
The First Sugar of Photosynthesis and the Rôle of Cane Sugar in the Plant.
}

DHotosynthesis, upon which all living organisms depend directly or indirectly for their existence, is still imperfectly understood. We know that the green plant takes in its carbon in the form of the carbon dioxide of the air, and we also know that carbohydrate appears in the leaf, as the result of the fixation of the carbon through the action of the sun's rays with the chlorophyll. The intermediate stages are still a matter of conjecture.

The formaldehyde hypothesis of Baeyer put forward so long ago as 1870 still awaits clear proof. Supposed conclusive evidence in its support has from time to time been advanced; but on critical examination has proved unacceptable. This has happened in a recent attempt to establish the validity of the theory. Klein and Werner ${ }^{1}$ using the delicate test of dimedon for aldehyde were in a measure convincing. Barton-Wright and Pratt, ${ }^{2}$ however, in repeating and checking their work, now show that the formaldehyde detected in the experiments is due to the action of light on carbon dioxide and bicarbonates, and is independent of the photosynthetic mechanism of the living plant. They sum up the matter thus: "Although the formaldehyde hypothesis has the merit of simplicity, which has probably been the prime cause of its wide popularity, no work has as yet convincingly shown that formaldehyde is produced normally in the green leaf or that it plays any part in the photosynthetic process of the living plant".

This statement is perhaps a little too sweeping. As formaldehyde is toxic to the plant, it is scarcely to be expected that it should accumulate in the free state in an appreciable amount under normal conditions. It may be assumed that, as soon as the reduction of the carbonic acid takes place, the aldehyde formed is at once polymerised to sugar.

The important work of Baly on photosynthesis in vitro is here relevant. $\mathrm{He}$ has shown recently ${ }^{3}$ that when a solution of carbon dioxide is exposed to ultra-violet light, formaldehyde is first formed and then sugar. But when a coloured catalyst, such as cobalt or nickel carbonate, is present, the action takes place in ordinary light with the direct production of sugar, no aldehyde being detected. Something analogous may therefore take place in the green leaf. The magnesium of the chlorophyll may be the active metal concerned. Hence on such a supposition it will be futile to search for free aldehyde in assimilating leaves.

In respect of the generally accepted view that some form of carbohydrate is the final product of carbon assimilation, the question as to the exact nature of this is still not completely answered. The classical experiments of Sachs conducted from 1862 onwards proved conclusively that the starch grains, which appear on the chloroplasts of the leaf when exposed to light, are the direct result of the fixation of carbon. Starch thus became known as the first visible product of assimilation. It was assumed that sugar, probably glucose, preceded the formation of starch.
Brown and Morris were the first to undertake elaborate estimations of the carbohydrates contained in foliage leaves. From their investigation of the carbohydrates in the leaf of the garden nasturtium (Tropoeolum majus), ${ }^{4}$ they concluded that eane sugar (sucrose) is the first earbohydrate to be set free in photosynthesis. This was an unexpected and novel idea, as sucrose, being a disaccharide, had hitherto been regarded more in the light of a reserve carbohydrate. They essayed a formidable task-a pioneer effort to estimate separately in the leaf four sugars (sucrose, maltose, dextrose, and levulose) as well as starch. The hydrolytic product of the starch complicated the issue. Monocotyledons, as a whole, in contrast to dicotyledons, form little starch in their leaves in normal circumstances, and some none at all. In following up Brown and Morris's work, Parkin, in order to simplify the problem, chose one of the latter, namely, the snowdrop. ${ }^{5}$ He found he had only three earbohydrates with which to deal, namely, sucrose and its two hexose derivatives, dextrose and levulose. He interpreted his results likewise in favour of sucrose being the first sugar liberated, and he regarded the two hexoses as arising from it through inversion.

The Rothamsted workers, Davis, Daish, and Sawyer, were the next to take up the subject. They advanced this side of biochemistry considerably by pointing out weaknesses in former methods and by elaborating new ones of extraction and analysis. They investigated in detail the carbohydrates of the mangold and potato leaf, ${ }^{6}$ and in their conclusions also support the view that sucrose is the first sugar of photosynthesis.

Now the pendulum is swinging the other way. Two important papers, by Clements ${ }^{7}$ and by Barton-Wright and Pratt, ${ }^{8}$ published last year, both favour the view that glucose (dextrose) is the first sugar to be liberated-a view, of course, more in harmony with Baeyer's hypothesis and also with chemistry generally. These investigators have carried out estimations at hourly intervals during both day and night--an advance upon what has been previously attempted.

Clements, an American worker, took for his research the leaves of the sunflower, the potato, and Soja max. Barton-Wright and Pratt, of Great Britain, selected the daffodil, which normally does not produce starch in its leaf. Their results are therefore comparable with those of Parkin on the snowdrop. In some respents they confirm the latter's work, though as regards the first sugar of photosynthesis they argue cogently for the opposite view.

Methods of analysis are naturally of prime importance in work of this kind. Each fresh investigator generally manages to bring to light flaws in previous methods. The Rothamsted workers with all their care are shown by BartonWright and Pratt to have overlooked a point which may have seriously prejudiced their figures for the hexose sugars. They find that the alcoholic 
extraction method used by Davis, Daish, and Sawyer results in the formation of appreciable amounts of aldehyde, and this affects considerably both the cupric reduction and the optical rotation.

Such work as has been quoted sheds no light on the exact position of the various sugars in the leaf. Strakosch some years ago applied certain microchemical tests to the assimilating leaf, and found hexose as the only sugar present in the palisade cells; but the reliability of his method is open to question. The work of Weevers on variegated leaves, published in 1924, is probably of greater value in support of the glucose view. He found both hexose sugars and sucrose in the green parts, but only sucrose in the white portions of the leaf.

The balance of evidence at the present time would seem to be in favour of the original supposition that glucose is the first sugar to be set free in photosynthesis. On the alternative view, the two hexose sugars are easily accounted for as the inversion products of the sucrose. But on the assumption that glucose appears first, then one has to imagine that part of it is first changed to fructose (levulose), and that from these two hexoses sucrose is synthesised. If these transformations do take place in the leaf, we are at present quite ignorant as to the means by which they are brought about.

Whatever may be the carbohydrate sequence in photosynthesis, there is cumulative evidence pointing to cane sugar as of wide, if not of universal, distribution among the higher plants. Apparently it is of fundamental importance, since no matter what form the carbohydrate may take in the seed or reserve organ, sucrose soon makes its appearance

on germination. The evidence is increasing that carbohydrate can travel largely in this form. Mason and Maskell's important work on the cotton plant ${ }^{9}$ favours this. Then, again, as a rule, leaves when fed with sugar solutions form starch more readily from sucrose than from any other sugar. Notwithstanding this apparent desire of the plant to have its carbohydrate in the form of sucrose for circulatory and metabolic purposes, there is no important reserve carbohydrate which yields cane sugar directly by enzymatic action!

In view of the fact that starch, the commonest of reserve carbohydrates, yields maltose when acted upon by its enzyme, diastase, this disaccharide rather than sucrose might have been expected to be chiefly evident in plant tissues; but it is not so. It may be that a non-reducing disaccharide is desired. Maltose has reducing properties, but sucrose has not. Then it has been suggested that the two hexose sugars, dextrose and levulose, which arise from the sucrose through inversion, may play different rôles in metabolism. Further, it is possible that these hexoses are more active when in the nascent state, that is, at the moment of their formation from sucrose through hydrolysis. This would account for cane sugar being found in meristematic tissue, upon which Priestley ${ }^{10}$ laid stress a few years ago.

J. P.

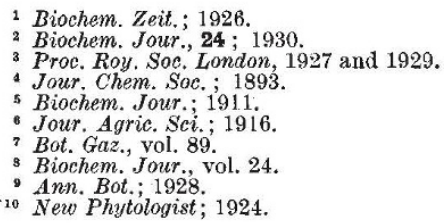

\section{Obituary.}

Prof. Hugh Ryan.

$\mathrm{B}^{\mathrm{Y}}$ $Y$ the death on Mar. 27 of Prof. Hugh Ryan at the age of fifty-eight, Irish chemists have sustained a loss which they will feel for many years to come.

Hugh Ryan was educated at Blackrock College, Dublin, and received his earlier chemical training under Prof. A. Senier in Queen's College, Galway. Graduating in 1895 , he obtained the M.A. degree, with gold medal, in 1897, and then proceeded to Berlin, where he engaged in research under Emil Fischer and Siegmund Gabriel. Returning to Dublin in 1899, he was appointed professor of chemistry in the Catholic University School of Medicine and in University College, St. Stephen's Green, Dublin. In the same year he was awarded the D.Sc. degree and appointed fellow of the Royal University of Ireland. On the foundation of the National University of Ireland in 1908, Ryan became professor of chemistry in University College, Dublin, a position which he occupied until his death. In 1924 he was appointed Chief State Chemist in the Irish Free State and was responsible for the organisation and control of the State Laboratory.

Throughout the entire period of his association with university teaching, Ryan devoted a large part of his time to research and published more than seventy papers. In his earlier work, influenced by his period with Fischer, he was engaged on the synthesis of glucosides. His researches in this direction, carried out under the most discouraging conditions, display his extraordinary enthusiasm and skill as a chemist. With the erection of the new buildings of University College, Dublin, under the Irish Universities Act of 1908, he had, for the first time, adequate laboratory equip. ment, and his remarkable powers as an organic chemist were given fuller scope. There followed a series of researches on the constitution of certain waxes and the preparation of a number of compounds allied in character to the colouring matter of turmeric. Further papers dealt with the synthesis of natural organic colouring matters and the preparation of derivatives of diflavone, diflavanone, and dicoumaranone. At the request of Nobel's Explosives Company, he undertook investigations on the mode of action of stabilisers in propellent explosives, the results of which were of the greatest value. Numerous other papers discussed the condensation of aldehydes with ketones and the structure of catechin. Ryan's many activities included a profound interest in Irish peat industries, and on this subject he furnished a very complete report to the Royal Dublin Society.

No. 3208 , VoL. 127] 